Sonderdruck aus

\title{
Archiv der Pharmazie
}

\author{
312. Band, Heft 5, Seite 385-389 \\ Verlag Chemie, GmbH, Weinheim (Bergstr.)
}

Stereoselektive Synthese des R-(+)-3-Phenyl-isochromans

Erwin v. Angerer und Wolfgang Wiegrebe 


\title{
Stereoselektive Synthese des R-(+)-3-Phenyl-isochromans
}

\author{
Erwin v. Angerer und Wolfgang Wiegrebe*)**)
}

Institut für Pharmazie, Lehrstuhl Pharmaz. Chemie I, der Universität Regensburg, Postfach 397, 8400 Regensburg 2.

Eingegangen am 31. Mai 1978

Die Synthese der Titelverbindung R-(+)-7 geht von L-(+)-Mandelsäure aus, die mit o-Brombenzylbromid zu 4 verethert wird. Ringschluß mit n-Butyllithium fuhrt zum Phenylisochromanon 5, das entspr. Carbinol-acetat $6 c$ wird zu 7 hydrogenolytisch gespalten.

\section{Stereoselective Synthesis of $(R)-(+)-3-P h e n y l i s o c h r o m a n e$}

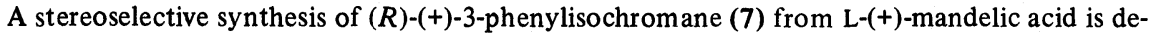
scribed. Condensation with $o$-bromobenzyl bromide leads to 4 , ring closure with n-butyllithium produces the phenylisochromanone 5, hydrogenolytic fission of the corresponding carbinol acetate $6 \mathrm{c}$ gives the desired compound 7 .

Im Zuge unserer Arbeiten zur Umsetzung von 1-[o-( $\alpha$-Hydroxyalkyl)-benzyl]-2-methyl1,2,3,4-tetrahydroisochinolinen 1 zu 3-Phenylisochromanen $2^{1)}$ (Abb. 1) haben wir u.a. die absol. Konfiguration des Eduktes $\mathbf{1 b}$ und die opt. Reinheit des Produktes 2b bestimmt ${ }^{2}$. Im Zusammenhang damit beschreiben wir in der vorliegenden Publikation die stereoselektive Synthese des unsubstituierten Grundkörpers 7.

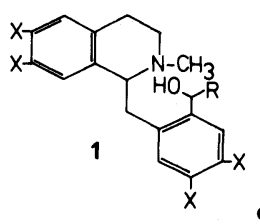

$\mathrm{X}=\mathrm{OCH}_{3}$

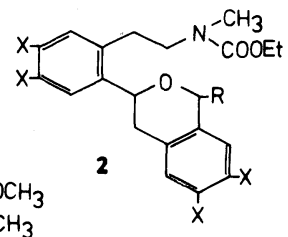

Abb. 1

a: $\mathrm{R}=\mathrm{CH}_{3}$

b: $R=H$

In Vorversuchen wurden verschiedene Vorstufen (s. Abb. 2 und Exp. Teil) auf ihre Eignung für unterschiedliche Ringschlußreaktionen untersucht. 3b liefert mit Friedel-Crafts-Reagentien Styrol. Beim Versuch einer reduktiven Kupplung von 3f 'urde der Benzylether gespalten. - In den Produktgemischen, die beim Behandeln des Tosylates 3k mit verschiedenen Basen entstanden, ließ sich kein 7-Hydroxy-3-phenylisochroman nachweisen. Nach diesen Versuchen an opt. inaktiven Mandelsäureethern ließ sich das Dinatriumsalz der L-(+)-Mandelsäure nicht mit 3,4-Dimethoxybenzylbromid umsetzen, die Umsetzung des L-(+)-Mandelsäuremethylesters mit diesem Benzylbromid und $\mathrm{Ag}_{2} \mathrm{O}^{3}$ ) schlug fehl, und die Williamson-Ethersynthese mit 3,4-Dimethoxybenzyl-

\footnotetext{
** Herrn Prof. Dr. C.H. Brieskorn zum 65. Geburtstag gewidmet
} 
bromid und dem O-Anion des L-(+)-Mandelsäuremethylesters führte zum Racemat des gewünschten Ethers 3g. Daraus folgte, daß eine stereoselektive Synthese von 7 entweder über das Anion der L-(+)-Mandelsäure oder über das zugehörige Carbinol bzw. dessen Derivate zu führen war. Saure Reaktionsbedingungen schieden nach den Vorversuchen aus.
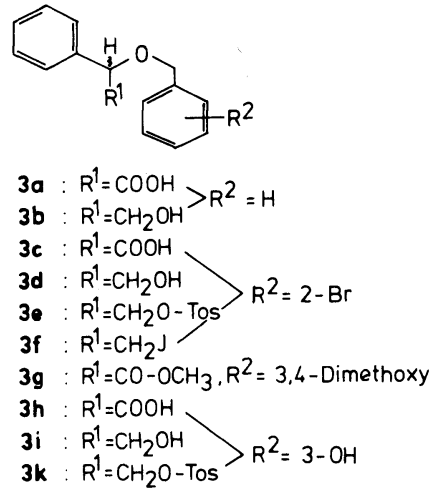

Abb. 2

Die Umsetzung der Carbonsäure 4 mit 2 Mol n-Butyllithium ${ }^{4}$ ) liefert opt. aktives 5 in 10-20proz. Ausbeute. Der Grad an opt. Reinheit ist bei 5 aller Voraussicht nach gering, doch sind opt. aktive Benzoine bekannt ${ }^{5)}$. Die Reduktion von 5 mit $\mathrm{LiAlH}_{4}$ liefert 6a erwartungsgemäß als Diastereomerengemisch, das als Gemisch der Tosylate 6b mit $\mathrm{LiAlH}_{4}$ umgesetzt wurde: Das Produktgemisch war optisch inaktiv und enthielt Carbonylverbindungen, vermutlich als Folge einer basenkatalysierten Eliminierung zum Enolether und dessen Spaltung beim Aufarbeiten unter Hydrolyse-Bedingungen. Nach Acetylierung von 6a zu 6c wurde deshalb mit $\mathrm{Pd} / \mathrm{BaSO}_{4}$ als Katalysator hydriert und 7 mit einer Restaktivität $[\alpha]_{\mathrm{D}}^{20}=+10^{\circ}$ erhalten. Die Struktur wurde durch Vergleich mit authentischem Racemat ${ }^{6}$ ) bewiesen.

Da das Chiralitätszentrum bei keinem Reaktionsschritt angegriffen wurde, überwiegt in 7 das Enantiomer mit R-Konfiguration. Bisher konnte 7 wegen der partiellen Racemisierung von $\mathbf{5}$ nicht optisch rein erhalten werden.
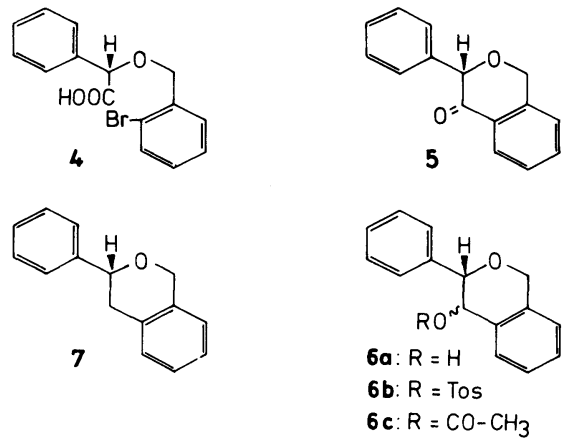

Abb. 3 


\section{Experimenteller Teil}

\section{Racemische Dibenzylether}

\subsection{3,4-Dimethoxybenzylbromid}

Abweichend von der Lit. Vorschrift 7) werden 3,36 g (20 mmol) Veratrylalkohol in $50 \mathrm{ml}$ absol. Ether gelöst und unter Feuchtigkeitsausschluß bei $-10^{\circ}$ mit $2,0 \mathrm{~g} \mathrm{PBr}_{3}$ versetzt. Man rührt je eine $\mathrm{h}$ bei $-10^{\circ}, 0^{\circ}$ und Raumtemp., gießt auf Eis und extrahiert zweimal mit je $20 \mathrm{ml}$ Wasser. Nach Trocknen $\left(\mathrm{MgSO}_{4}\right)$ und Abziehen des Ethers erhält man 3,80 g reines, kristallines Produkt. Schmp. $56^{\circ}$ (Petrolether 50-70 $)$. Lit.: 7): 59 ${ }^{\circ}$. ${ }_{9} \mathrm{H}_{11} \mathrm{BrO}_{2}$ (232,1); Ber.: C 46,6, H 4,80; Gef.: $\mathrm{C} 47,6, \mathrm{H} \mathrm{4,64.}{ }^{1} \mathrm{H}-\mathrm{NMR}\left(\mathrm{CDCl}_{3}\right): \delta$ (ppm) $=6.90$ (verbreitert. $\mathrm{s} ; 2 \mathrm{H}$ aromat.), 6.80 (verbreitert. s; $1 \mathrm{H}$ aromat.), 4.47 (s; $\left.2 \mathrm{H}, \mathrm{CH}_{2} \mathrm{Br}\right), 3.87\left(\mathrm{~s} ; 3 \mathrm{H}, \mathrm{OCH}_{3}\right), 3.84\left(\mathrm{~s} ; 3 \mathrm{H}, \mathrm{OCH}_{3}\right)$.

\subsection{2-Phenyl-2-benzyloxy-ethanol (3b)}

3a 3) wird mit $\mathrm{LiAlH}_{4}$ in Ether bei Raumtemp. reduziert. Reinigung durch Chromatographie an Kieselgel 60 (Merck) mit Ether/Methylenchlorid (1:1); Ausb.: $86 \%$ eines farblosen Öls. ${ }^{1} \mathrm{H}-\mathrm{NMR}$ $\left(\mathrm{CDCl}_{3}\right): \delta(\mathrm{ppm})=7.34$ (s; $5 \mathrm{H}$ aromat.), 7.30 (s; $5 \mathrm{H}$ aromat.), $4.46\left(\mathrm{~m} ; 3 \mathrm{H} \mathrm{Ar}-\mathrm{C}_{2}-\mathrm{O},>\mathrm{CH}-\mathrm{O}\right)$, $3.63\left(\mathrm{~m}, 2 \mathrm{H} \mathrm{C}_{2}-\mathrm{OH}\right), 2,67$ (s; $\left.1 \mathrm{H} \mathrm{OH}\right)$.

\subsection{Phenyl-(2-brom-benzyloxy)-essigsäure (3c)}

$6,1 \mathrm{~g}$ ( $36 \mathrm{mmol}$ ) Mandelsäuremethylester in $70 \mathrm{ml}$ absol. Dioxan werden mit $30 \mathrm{~g}$ o-Brombenzylbromid, $20 \mathrm{~g} \mathrm{Ag}_{2} \mathrm{O}$ und $10 \mathrm{~g} \mathrm{MgSO}_{4}$ versetzt und $15 \mathrm{~h}$ unter Rückfluß gekocht. Nach dem $\mathrm{Ab}$ kühlen saugt man ab, wäscht mit $100 \mathrm{ml}$ Dioxan nach, gibt $60 \mathrm{ml}$ Wasser und 3,5 $\mathrm{ml} \mathrm{konz}$. $\mathrm{H}_{2} \mathrm{SO}_{4}$ zu und erhitzt $20 \mathrm{~h}$ zum Sieden. Man versetzt mit $2 \mathrm{~N} \mathrm{NaOH}$ bis zur alkalischen Reaktion, extrahiert mit Methylenchlorid, säuert an und schüttelt wieder mit Methylenchlorid aus. Der Extrakt enthält 12,0 g eines Öls, aus dem durch Kristallisation aus Petrolether 9,7 g 3c als farblose Kristalle erhalten werden. Ausb.: $83 \%$, Schmp. 87-88 ${ }^{\circ} \mathrm{C}_{15} \mathrm{H}_{13} \mathrm{BrO}_{3}(321,3)$; Ber.: C 56,1, H 4,08; Gef.: C 56,1, H 3,67. ${ }^{1} \mathrm{H}-\mathrm{NMR}\left(\mathrm{CDCl}_{3}\right): \delta(\mathrm{ppm})=11.37$ (s; $\left.1 \mathrm{H} \mathrm{COOH}\right), 7.40$ (m; $9 \mathrm{H}$ aromat.), 5.00 (s; $1 \mathrm{H}>\mathrm{CH}-\mathrm{O}), 4.66$ (s; $\left.\mathrm{Ar}-\underline{\mathrm{C}}_{2}-\mathrm{O}\right)$.

\subsection{2-Phenyl-2-(2-brom-benzyloxy)-ethanol (3d)}

Reduktion von 3c mit $\mathrm{LiAlH}_{4}$ in Ether. Ausb.: $90 \%$ farbloses Öl. ${ }^{1} \mathrm{H}-\mathrm{NMR}\left(\mathrm{CDCl}_{3}\right): \delta(\mathrm{ppm})=$ 7.33 (m; $9 \mathrm{H}$ aromat.), 4.50 (m; $\left.3 \mathrm{H} \mathrm{Ar}-\underline{\mathrm{CH}}_{2}-\mathrm{O}+>\mathrm{CH}-\mathrm{O}\right), 3.67$ (m;2 $\left.\mathrm{H} \mathrm{C} \underline{H}_{2}-\mathrm{OH}\right), 2.70$ ( $; 11 \mathrm{H}$ $\mathrm{OH})$.

\subsection{Toluolsulfonsäureester von $3 \mathrm{~d}(3 \mathrm{e})$}

Tosylierung nach Organikum $\left.{ }^{8}\right)$. Ausb.: $46 \%$, Schmp. 81-82 ${ }^{\circ}$ (Ethanol). ${ }^{1} \mathrm{H}-\mathrm{NMR}\left(\mathrm{CDCl}_{3}\right)$ : $\delta(\mathrm{ppm})=7.77(\mathrm{~d} ; \mathrm{J}=8 \mathrm{~Hz}, 2 \mathrm{H}$ aromat. $), 7.33$ (m;11 $\mathrm{H}$ aromat. $), 4.63(\mathrm{~m} ; 1 \mathrm{H}>\mathrm{CH}-\mathrm{O}), 4.43$ (s; 2 H Ar-C $\underline{H}_{2}-\mathrm{O}$ ), 4.20 (m; $\left.2 \mathrm{H} \mathrm{C}_{2}-\mathrm{O}-\mathrm{Tos}\right), 2.40$ (s; $3 \mathrm{H} \mathrm{CH}_{3}$ ).

\subsection{1-Phenyl-1-(2-brom-benzyloxy)-2-jod-ethan (3f)}

$3,30 \mathrm{~g} \mathrm{(} 7 \mathrm{mmol}) 3 \mathrm{e}$ und 1,6 $\mathrm{g} \mathrm{NaJ}$ werden in $30 \mathrm{ml}$ Aceton $50 \mathrm{~h}$ zum Sieden erhitzt. Nach Eindampfen i. Vak. nimmt man in Ether auf und entfernt das Solvens i. Vak. Der Rückstand wird an Kieselgel 60 (Merck) chromatographiert (Methylenchlorid). Ausb.: $45 \%$, Sdp. $0,1{ }_{1} 150^{\circ} .{ }^{1} \mathrm{H}-$ $\operatorname{NMR}\left(\mathrm{CDCl}_{3}\right): \delta(\mathrm{ppm})=7.35(\mathrm{~m} ; 9 \mathrm{H}$ aromat. $), 4.50\left(\mathrm{~m} ; 3 \mathrm{H}>\mathrm{CH}-\mathrm{O}+\mathrm{Ar}_{-} \underline{\mathrm{H}}_{2}-\mathrm{O}\right), 3.33(\mathrm{~m}$; $2 \mathrm{H} \mathrm{CH}_{2} \mathrm{~J}$ ). 


\subsection{Phenyl-(3,4-dimethoxy-benzyloxy)-essigsäuremethylester (3g)}

$1,68 \mathrm{~g}(10 \mathrm{mmol})$ Mandelsäuremethylester in $10 \mathrm{ml}$ absol. Ether werden bei $0^{\circ}$ in eine Suspension von $300 \mathrm{mg} \mathrm{NaH}$ in $20 \mathrm{ml}$ absol. Ether getropft. Nach Bildung eines Niederschlages gibt man 2,33 $\mathrm{g}$ (10 mmol) 3,4-Dimethoxybenzylbromid in $15 \mathrm{ml}$ Ether zu und erhitzt $3 \mathrm{~h}$ zum Sieden. Man ersetzt den Ether durch $30 \mathrm{ml}$ Dioxan, kocht über Nacht unter Rückfluß, gießt in $150 \mathrm{ml}$ Wasser und extrahiert mit Ether. Das so erhaltene farblose Öl wird durch Chromatographie an $\mathrm{Al}_{2} \mathrm{O}_{3}$ (Ak tivitätsstufe II-III) mit Ether gereinigt: $1,10 \mathrm{~g}(35 \%) 3 \mathrm{~g} .{ }^{1} \mathrm{H}-\mathrm{NMR}\left(\mathrm{CDCl}_{3}\right): \delta(\mathrm{ppm})$ $=7.37$ (m; $5 \mathrm{H}$ aromat.), 6.87 (m; $3 \mathrm{H}$ aromat. $), 4.90(\mathrm{~s} ; 1 \mathrm{H}>\mathrm{CH}-\mathrm{O}), 4.50\left(\mathrm{~s} ; 2 \mathrm{H} \mathrm{Ar}-\mathrm{CH}_{2}-\mathrm{O}\right)$, $3.83\left(\mathrm{~s} ; 6 \mathrm{H} \mathrm{OCH}_{3}\right), 3.67\left(\mathrm{~s} ; 3 \mathrm{H} \mathrm{COOCH}_{3}\right)$.

\subsection{Phenyl-(3-hydroxy-benzyloxy)-essigsäure (3h)}

$2,60 \mathrm{~g}$ (15 mmol) Mandelsäuremethylester werden in $30 \mathrm{ml}$ absol. Dioxan gelöst und mit $25 \mathrm{~g}$ m-Acetoxybenzylbromid, $10 \mathrm{~g} \mathrm{Ag}_{2} \mathrm{O}$ und $5 \mathrm{~g}$ wasserfreiem $\mathrm{MgSO}_{4}$ versetzt. Unter kräftigem Rühren kocht man $15 \mathrm{~h}$ unter Rückfluß. Nach dem Abkühlen saugt man ab, wäscht mit $100 \mathrm{ml}$ Dioxan nach, fugt $40 \mathrm{ml}$ Wasser und $2,0 \mathrm{ml}$ konz. $\mathrm{H}_{2} \mathrm{SO}_{4} \mathrm{zu}$ und erhitzt $6 \mathrm{~h}$ zum Sieden. Nach Zugabe von weiteren $100 \mathrm{ml}$ Wasser extrahiert man mit Methylenchlorid. Die organische Phase wird mit $\mathrm{NaHCO}_{3}$-Lösung ausgeschüttelt; nach Ansäuern und Extraktion mit Methylenchlorid erhält man $3,3 \mathrm{~g}(82 \%) 3 \mathrm{~h}$ als gelbes Öl. ${ }^{1} \mathrm{H}-\mathrm{NMR}\left(\mathrm{CDCl}_{3}\right): \delta(\mathrm{ppm})=9.00(\mathrm{~s} ; 2 \mathrm{H} \mathrm{OH}+\mathrm{COOH})$, 7.30 (m; $5 \mathrm{H}$ aromat.), 6.80 (m; $4 \mathrm{H}$ aromat.), 4.90 (s; $1 \mathrm{H}>\mathrm{CH}-\mathrm{O}$ ), 4.43 (s; $2 \mathrm{H} \mathrm{Ar}-\underline{\mathrm{H}}_{2}-\mathrm{O}$ ).

\subsection{2-Phenyl-2-(3-hydroxy-benzyloxy)-ethanol '3i)}

Reduktion von $3 \mathrm{~h}$ mit einem 30proz. Überschuß an $\mathrm{LiAlH}_{4}$ in Ether: farbloses Öl, das kristallin erstarrt. Schmp.: $101-103^{\circ}$ (Benzol/Petrolether). $\mathrm{C}_{15} \mathrm{H}_{16} \mathrm{O}_{3}(244,3)$; Ber.: C 73,8, $\mathrm{H}$ 6,60; Gef.: $\mathrm{C} \mathrm{73,9,} \mathrm{H} \mathrm{6,48.}{ }^{1} \mathrm{H}-\mathrm{NMR}\left(\mathrm{CDCl}_{3}\right): \delta(\mathrm{ppm})=7.33$ ( $; 55 \mathrm{H}$ aromat.), 6.83 (m; $4 \mathrm{H}$ aromat.), 6.75 (s; $2 \mathrm{H} 2 \times \mathrm{OH}$ ), 4.55 (dd; J = 5/7 Hz, $1 \mathrm{H}>\mathrm{CH}-\mathrm{O}$ ), 4.53 (d; J = $12 \mathrm{~Hz}, 1 \mathrm{H} \mathrm{Ar}-\mathrm{CH}_{2}-\mathrm{O}$ ), 4.23 (d; $\left.\mathrm{J}=12 \mathrm{~Hz}, 1 \mathrm{H} \mathrm{Ar}-\underline{\mathrm{H}}_{2}-\mathrm{O}\right), 3.74\left(\mathrm{~d} ; \mathrm{J}=7 \mathrm{~Hz}, 1 \mathrm{H} \mathrm{C}_{2}-\mathrm{OH}\right), 3.70\left(\mathrm{~d} ; \mathrm{J}=5 \mathrm{~Hz}, 1 \mathrm{H} \mathrm{C}_{2}-\mathrm{OH}\right)$.

\subsection{0. p-Toluolsulfonsäureester von $3 \mathbf{i}(3 \mathbf{k})$}

Tosylierung nach Organikum ${ }^{8}$ ). Reinigung durch Chromatographie an Kieselgel 60 (Merck) (Methylenchlorid/Ether 1:1): Ausb.: $70 \%$ farbloses, kristallisierendes Öl, Schmp. 108-110 , das ohne Reinigung weiterverarbeitet wurde. ${ }^{1} \mathrm{H}-\mathrm{NMR}\left(\mathrm{CDCl}_{3}\right): \delta(\mathrm{ppm})=7.67(\mathrm{~d} ; \mathrm{J}=8 \mathrm{~Hz}, 2 \mathrm{H}$ aromat.), 7.07 (d; J = $8 \mathrm{~Hz}, 2 \mathrm{H}$ aromat.), 7.27 (s; $5 \mathrm{H}$ aromat.), 6.83 (m; $4 \mathrm{H}$ aromat.), 5.50 (s; $1 \mathrm{H} \mathrm{OH}$ ), 4.63 (dd; J = 7/5 Hz, $1 \mathrm{H}>\mathrm{CH}-\mathrm{O}), 4.52$ (d; J = $\left.12 \mathrm{~Hz}, 1 \mathrm{H} \mathrm{Ar}-\mathrm{CH}_{2}-\mathrm{O}\right), 4.20$ (d; J = $12 \mathrm{~Hz}, 1 \mathrm{H} \mathrm{Ar}-\underline{\mathrm{C}}_{2}-\mathrm{O}$ ), 4.13 (d; $\left.\mathrm{J}=7 \mathrm{~Hz}, 1 \mathrm{H} \mathrm{C}_{2}-\mathrm{O}-\mathrm{Tos}\right), 4.10$ (d; J = $5 \mathrm{~Hz}, 1 \mathrm{H} \mathrm{CH}_{2}$-O-Tos), $2.37\left(s ; 3 \mathrm{H} \mathrm{CH}_{3}\right)$.

\section{Umsetzung mit opt. aktiven Mandelsäure-Derivaten}

\subsection{S-(+)-Phenyl-(2-brom-benzyloxy)-essigsäure (4)}

Analog 1.3. Ausb.: $85 \%$, Schmp. 97-98 (Petrolether), $[\alpha]_{\mathrm{D}}^{20}=+76^{\circ} . \mathrm{C}_{15} \mathrm{H}_{13} \mathrm{BrO}_{3}(321,3)$; Ber.: C 56,1; H 4,08, Br 24,9; Gef.: C 55,9, H 4,12, Br 25,4.

\subsection{S-(+)-3-Phenylisochromanon-4 (5)}

$2,0 \mathrm{~g}(6,6 \mathrm{mmol}) 4$ werden unter $\mathrm{N}_{2}$ in $100 \mathrm{ml}$ absol. THF gelöst. Bei $-70^{\circ}$ tropft man $8,5 \mathrm{ml}$ Butyllithium-Lösung (15 proz.) hinzu, rührt $60 \mathrm{~min}$. bei $-70^{\circ}$ und je $30 \mathrm{~min}$. bei $-55^{\circ},-45^{\circ}$, $-35^{\circ}$ und $-25^{\circ}$, fügt $5 \mathrm{ml}$ Wasser zu und engt i.Vak. auf $15 \mathrm{ml}$ ein. Die verbleibende Lösung gibt man 
zu $100 \mathrm{ml}$ Wasser und $100 \mathrm{ml}$ Ether und wäscht die organische Phase neutral. Nach Trocknen und Abziehen des Solvens verbleiben $270 \mathrm{mg}$ eines farblosen Öles $(19 \%),[\alpha]_{\mathrm{D}}^{20}=+11^{\circ}\left(\mathrm{CDCl}_{3}\right)$. IR: $1695 \mathrm{~cm}^{-1}$ (Film). ${ }^{1} \mathrm{H}-\mathrm{NMR}$ identisch mit (士)-3-Phenylisochromanon-4 9).

\subsection{S-(+)-4-Hydroxy-3-phenylisochromane (6a) (Diastereomeren-Gemisch)}

$250 \mathrm{mg}$ Phenylisochromanon $5(1,1 \mathrm{mmol})$ in absol. Ether werden mit überschüssigem $\mathrm{LiAlH}_{4}$ versetzt und $3 \mathrm{~h}$ bei Raumtemp. gerührt. Nach üblicher Aufarbeitung verbleiben $190 \mathrm{mg}$ schmieriger Kristalle. Chromatographie mit $\mathrm{CH}_{2} \mathrm{Cl}_{2}$ an Kieselgel 60 (Merck) liefert $110 \mathrm{mg} \mathrm{(44 \% )} \mathrm{farb-}$ lose Kristalle. Schmp. $88-89^{\circ}$ (Cyclohexan). $[\alpha]_{\mathrm{D}}^{20}=+46^{\circ}\left(\mathrm{CDCl}_{3}\right){ }^{1}$ H-NMR identisch mit (+)-4-Hydroxy-3-phenylisochroman 9 ).

\subsection{S-1+)-4-Acetoxy-3-phenylisochromane (6c) (Diastereomeren-Gemisch)}

$270 \mathrm{mg}$ ( $1,2 \mathrm{mmol}) 6 \mathrm{a}, 1,5 \mathrm{mmol}$ Acetanhydrid und 1,5 mmol Pyridin werden in $4 \mathrm{ml}$ Chloroform $3 \mathrm{~h}$ unter Rückfluß gekocht. Nach dem Ausschütteln mit verd. $\mathrm{H}_{2} \mathrm{SO}_{4}$ und Wasser und $\mathrm{Ab}$ ziehen des Solvens erhält man ein farbloses Öl. Chromatographie an Kieselgel 60 (Merck) (Methylenchlorid/Ether 2:1) erbringt $180 \mathrm{mg}(56 \%)$ kristallines Produkt. Schmp. 112-115 (Ethanol); $[\alpha]_{\mathrm{D}}^{20}=+32^{\circ}\left(\mathrm{CHCl}_{3}\right) \cdot \mathrm{C}_{17} \mathrm{H}_{16} \mathrm{O}_{3}(268,3)$; Ber.: C 76,1, $\mathrm{H} \mathrm{6,01}$; Gef.: 76,0, $\mathrm{H} \mathrm{6,00.}{ }^{1} \mathrm{H}-\mathrm{NMR}$ $\left(\mathrm{CDCl}_{3}\right): \delta(\mathrm{ppm})=7.26(\mathrm{~m} ; 9 \mathrm{H}$ aromat. $), 6.13(\mathrm{~m} ; 1 \mathrm{H}>\mathrm{CH}-\mathrm{O}-\mathrm{Ac}), 4.90(\mathrm{~m} ; 3 \mathrm{H}>\mathrm{CH}-\mathrm{O}+$ $\mathrm{Ar}-\underline{\mathrm{C}}_{2}-\mathrm{O}$ ), 2.00 (s) +1.73 (s) (zusammen $3 \mathrm{H} \mathrm{CH}_{3}$ ).

\section{5. $R-(+)-3-P h e n y l i s o c h r o m a n(7)$}

$270 \mathrm{mg}(1 \mathrm{mmol}) 6 \mathrm{c}$ werden in $150 \mathrm{ml}$ Ethanol $(96 \%)$ suspendiert und nach Zugabe von $250 \mathrm{mg}$ Katalysator ( $10 \% \mathrm{Pd}$ auf $\left.\mathrm{BaSO}_{4}\right)$ in Wasserstoffatmosphäre $30 \mathrm{~min}$. bei $35^{\circ}$ und $3 \mathrm{~h}$ bei Raumtemp. unter Normaldruck geschüttelt. Nach Abziehen des Lösungsmittels unterwirft man den Rückstand der Chromatographie an Kieselgel 60 (Merck) (Methylenchlorid/Petrolether 1:1).

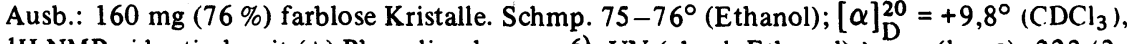
${ }^{1}$ H-NMR: identisch mit ( \pm )-Phenylisochroman 6$)$. UV (absol. Ethanol) $\lambda_{\max }(\log \epsilon): 223(3,18)$, 251 (2,52), $257(2,67), 263(2,71), 272(2,65)$ nm. $\mathrm{C}_{15} \mathrm{H}_{14} \mathrm{O}(210,3)$; Ber.: C 85,7, H 6,71; Gef.: C 85,2, H 6,70.

\section{Literatur}

1 W. Wiegrebe, Arch. Pharm. (Weinheim) 301, 672 (1968).

2 H.M. Stephan, G. Langer und W. Wiegrebe, Pharm. Acta Helv. 51, 164 (1976).

3 W.A. Bonner, J. Org. Chem. 32, 2496 (1967).

4 R.J. Boatman, B.J. Whitlock und H.W. Whitlock, Jr., J. Am. Chem. Soc. 99, 4822 (1977).

5 M. McKenzie und H. Wren, J. Chem. Soc. 93, 309 (1908).

6 R.L. Vaulx, F.N. Jones und C.R. Hauser, J. Org. Chem. 29, 1387 (1964).

7 K. Freudenberg, G. Carrara und E. Cohn, Justus Liebigs Ann. Chem. 446, 87 (1926).

8 Organikum, 14. Aufl., S. 608, VEB Deutscher Verlag der Wissenschaften, Berlin 1975.

9 C. Normant-Chefnay, Bull. Soc. Chim. Fr. 1971, 1351, 1362. 